\title{
Determination of sulfation pattern in brain glycosaminoglycans by chip-based electrospray ionization ion trap mass spectrometry
}

\author{
Corina Flangea - Catalin Schiopu • Eugen Sisu • \\ Alina Serb • Michael Przybylski • Daniela G. Seidler • \\ Alina D. Zamfir
}

Received: 29 June 2009/Revised: 15 September 2009/Accepted: 17 September 2009/Published online: 14 October 2009

\begin{abstract}
Chondroitin sulfate (CS) and dermatan sulfate (DS) glycosaminoglycans display variability of sulfation in their constituent disaccharide repeats during chain elongation. Since a large proportion of the extracellular matrix of the central nervous system (CNS) is composed of proteoglycans, CS/DS disaccharide degree and profile of sulfation play important roles in the functional diversity of neurons, brain development, and some of its pathological states. To investigate the sulfation pattern of CS/DS structures expressed in CNS, we introduced here a novel method based on an advanced system encompassing fully automated chip nanoelectrospray ionization (nanoESI) in the negative ion mode and high capacity ion trap multistage mass spectrometry $\left(\mathrm{MS}^{2}-\mathrm{MS}^{3}\right)$ by collision-induced dissociation (CID). This method, introduced here for the first time in glycomics of brain glycosaminoglycans, was particularly applied to structural investigation of disaccharides obtained by $\beta$ elimination and digestion with chondroitin $\mathrm{B}$ and $\mathrm{AC}$ I lyase
\end{abstract}

of hybrid CS/DS chains from wild-type mouse brain. Screening in the chip-MS mode of DS disaccharide fraction resulting after depolymerization with chondroitin $\mathrm{B}$ lyase revealed molecular ions assigned to monosulfated disaccharide species having a composition of 4,5- $\Delta$-[IdoA-GalNAc]. By optimized CID $\mathrm{MS}^{2}-\mathrm{MS}^{3}$, fragment ions supporting the localization of sulfate ester group at $\mathrm{C} 4$ within GalNAc were produced. Chip ESI MS profiling of CS disaccharide fraction obtained by depolymerization of the same CS/DS chain using chondroitin $\mathrm{AC} I$ lyase indicated the occurrence of mono- and bisulfated 4,5- $\triangle$-[GlcA-GalNAc]. The site of oversulfation was determined by $\mathrm{MS}^{2}-\mathrm{MS}^{3}$, which provided sequence patterns consistent with a rare GlcA-3-sulfateGalNAc-6-sulfate structural motif.

Keywords Chondroitin/dermatan sulfate - Mouse brain . Fully automated chip-based nanoelectrospray.

Ion trap mass spectrometry
C. Flangea $\cdot$ C. Schiopu $\cdot$ A. Serb $\cdot$ A. D. Zamfir $(\bowtie)$

Mass Spectrometry Laboratory, National Institute for Research and Development in Electrochemistry and Condensed Matter, Plautius Andronescu Str. 1, 300224 Timisoara, Romania e-mail: alina.zamfir@uav.ro

C. Flangea $\cdot$ E. Sisu $\cdot$ A. Serb

"Victor Babes" University of Medicine and Pharmacy,

Eftimie Murgu Square, 2,

300054 Timisoara, Romania

E. Sisu

Chemistry Institute of Romanian Academy,

Mihai Viteazul Blvd 24,

300223 Timisoara, Romania

\section{Przybylski}

Laboratory of Analytical Chemistry and Biopolymer Structure

Analysis, University of Konstanz,

Universitätsstrasse 10 ,

78464 Konstanz, Germany

\section{G. Seidler}

Institute for Physiological Chemistry and Pathobiochemistry,

University of Münster,

Waldayer Str. 15,

48149 Münster, Germany

\section{A. D. Zamfir}

Department of Chemical and Biological Sciences,

"Aurel Vlaicu" University of Arad,

Revolutiei Blvd 77,

310130 Arad, Romania

Konstanzer Online-Publikations-System (KOPS)

URN: http://nbn-resolving.de/urn:nbn:de:bsz:352-opus-109830

URL: http://kops.ub.uni-konstanz.de/volltexte/2010/10983 


\section{Introduction}

Extracellular matrix of central nervous system (CNS) serves as supporting structure for cells and rich source of signaling molecules that can influence cell proliferation, survival, migration, and differentiation. A large proportion of this matrix consists of proteoglycans (PGs) - proteins with long chains of covalently attached polysaccharides called glycosaminoglycans (GAGs) [1].

Chondroitin sulfate (CS) and dermatan sulfate (DS) glycosaminoglycans are covalently linked to a wide range of $\mathrm{PG}$ core proteins, with a widespread distribution in mammalian tissue. $\mathrm{CS}$ chain backbone encompasses repetitive disaccharide units containing D-glucuronic acid (GlcA) and $N$-acetylgalactosamine (GalNAc) residues. DS is the stereoisomeric variant of CS with varying proportions of $\mathrm{L}-$ iduronic acid (IdoA). CS/DS-PGs are involved in many biological processes [2], such as cell proliferation and recognition [3], cell adhesion and migration, neurite outgrowth [4], and wound repair [5]. In mammalian tissues, these chains are often found as hybrid CS/DS structures. Their further structural variability during elongation is produced by divergent sulfation in the repeating disaccharide units [6].

$\mathrm{CS}$, heparan sulfate (HS), and hyaluronic acid are GAGs ubiquitously expressed in neural tissue; however, the occurrence of keratan sulfate (KS) and DS was also reported [7]. Recent studies have shown that proportions of CS/DS disaccharides in brain change with development/ affliction, which suggests that CS/DS species differing in the degree and profile of sulfation are correlated with the functional diversity of neurons [8]. Oversulfated CS, DS, and hybrid CS/DS structures have been found strongly implicated in brain development [9]. Several reports point out that CS/DS chains of mammalian brains are characterized by an elevated degree of microheterogeneity and highly sulfated domains containing a cluster of oversulfated disaccharides [10]. Lately, a novel neurobiological function was attributed to oversulfated DS. In an experimental model on cultures of hippocampal neurons from E16 mouse brains [11], physiological neuritogenesis during brain development was unequivocally associated to oversulfated DS structures.

A prerequisite for collecting reliable information on brain proteoglycan glycosylation is the development of advanced analytical methods capable to offer data upon epimerization, sulfate content, and its distribution. The arsenal of currently available sophisticated mass spectrometric (MS) techniques opened new perspectives in this regard. Thoroughly optimized methods based on electrospray (ESI) MS were shown to provide accurate results on unsulfated and regularly and oversulfated domains in chondroitin and dermatan oligomers $[12,13]$. For CS/DS and also HS and KS, the incidence of regular and/or irregular sulfation pattern(s) was directly documented by sequencing single components in tandem MS (MS/MS) experiments by collision-induced [14-19] (CID) or electron detachment $[20,21]$ dissociation.

Due to its potential to reduce the in-source loss of labile attachments such as sulfate ester groups and increase analysis speed, sensitivity, and ionization efficiency, chipbased nanoelectrospray ionization (nanoESI) emerged recently as a valuable tool in glycosaminoglycomics [22-26].

Although MS demonstrated its ability to offer distinctive insights into the domain structure of GAGs; so far no structural analysis of CNS-derived GAGs was pursued by employing mass spectrometry-based methodologies.

To close this gap, here, mass spectrometry is for the first time introduced in glycomics of brain glycosaminoglycans and particularly applied to brain-associated CS/DS oligosaccharides. The newly developed MS method is based on fully automated chip nanoESI performed on a NanoMate 400 robot in direct coupling with a high capacity ion trap (HCT) mass spectrometer. CS and DS, obtained by $\beta$ elimination and selective digestion of hybrid chains from wild-type mouse brain, were screened and sequenced by NanoMate HCT MS operating in negative ion. mode. By this advanced MS protocol, we were able to identify brain DS repeats of regular sulfation pattern as well as an unusual bisulfated CS disaccharide. Moreover, sequencing in the multistage mass spectrometry $\left(\mathrm{MS}^{2}-\mathrm{MS}^{3}\right)$ mode gave rise to fragmentation patterns consistent with an atypical GlcA3-sulfate-GalNAc-6-sulfate motif.

\section{Experimental}

Materials and standards

Analytical grade methanol was purchased from Merck (Darmstadt, Germany) and used without further purification. All sample solutions were dried in a SpeedVac Concentrator, SPD 111V-230 from Thermo Electron Corporation, (Asheville, NC, USA), coupled to a vacuum pump PC 2002 Vario with CVC 2000 Controller from Vaccubrand (Wertheim, Germany). Prior to chip-based MS analysis, the sample/methanol solutions were centrifuged for $2 \mathrm{~h}$ in a SIGMA 2-16 model centrifuge from Sartorius GmbH (Göttingen, Germany).

\section{Mouse brain glycosaminoglycans}

Two C57BL/6 whole brains (total mass, $m=0.83 \mathrm{~g}$ ) of 4 week-old healthy mice were sampled. The option for this age was guided by the mouse growth spurt phase, which at the age of 4 weeks is completed. The brain tissue was 
Fig. 1 Fully automated chip (-) nanoESI HCT MS of brain DS disaccharide obtained after depolymerization with chondroitin B lyase and separation on GFCSuperdex peptide column. Solvent, MeOH; ESI MS parameters: ChipESI, $1.4 \mathrm{kV}$; Cap Exit, 50 V. Back nitrogen pressure 0.30 psi. Nitrogen nebullizer on MS at $50 \mathrm{psi}$

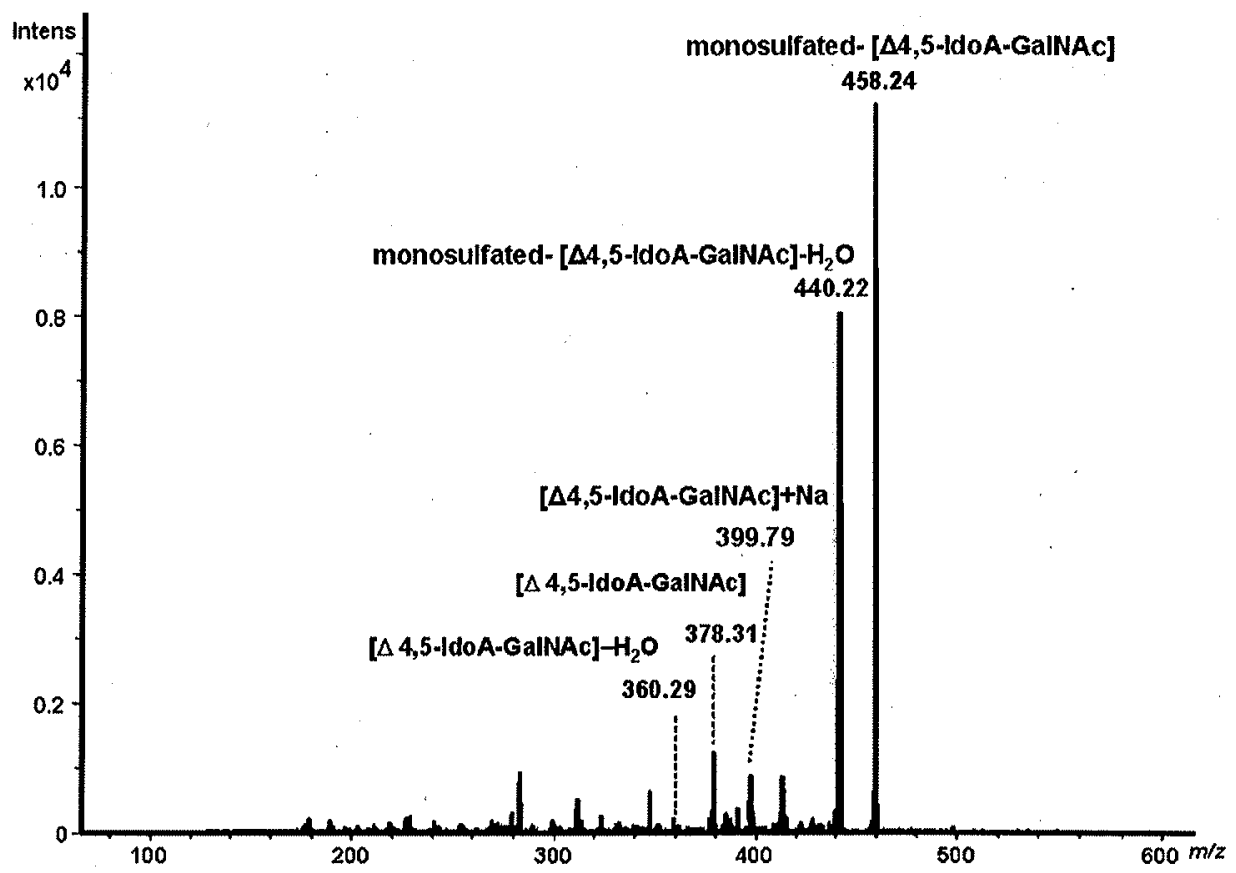

dissolved in $4 \mathrm{M}$ guanidinum chloride buffer and rotated over night at $4{ }^{\circ} \mathrm{C}$. For purification, the sample was centrifuged, and the supernatant was diluted with starting buffer $(20 \mathrm{mM}$ Tris/ $\mathrm{HCl} \mathrm{pH}$ 7.4) containing $150 \mathrm{mM} \mathrm{NaCl}$. This solution was applied on DEAE-Tris-Acryl $M$ prepared in a Pasteur pipette with a bedvolume of $0.5 \mathrm{~mL}$ and washed with $5 \times$ bedvolume starting buffer containing $150 \mathrm{mM} \mathrm{NaCl}$ followed by $300 \mathrm{mM} \mathrm{NaCl}$ [27]. The CS/DS proteoglycans were eluted with $3 \times$ bedvolume starting buffer containing
$1 \mathrm{M} \mathrm{NaCl}$, desalted and concentrated. One-half volume was digested with $50 \mathrm{mU} / \mathrm{assay}$ chondroitin $\mathrm{AC}$ I lyase in $200 \mu \mathrm{L} 50 \mathrm{mM}$ Tris $/ \mathrm{HCl}$, pH 8.0 , containing $60 \mathrm{mM}$ sodium acetate, $60 \mathrm{mM} \mathrm{NaCl}, 0.01 \%$ bovine serum albumin, and $3 \mathrm{mM} \mathrm{NaN}_{3}$ for $2 \mathrm{~h}$ at $37{ }^{\circ} \mathrm{C}$ (Seikagaku Kogyo, Tokyo, Japan) as described previously [27, 28]. In parallel, the other one-half volume was digested with $50 \mathrm{mU} /$ assay chondroitin B lyase. Size fractionation of oligosaccharides was performed on a Superdex Peptide HR10/30
Fig. 2 Fully automated chip (-) nanoESI HCT MS of brain CS disaccharide obtained after depolymerization with chondroitin AC I lyase and separation on GFC-Superdex peptide column. Experiment conditions as in Fig. 1

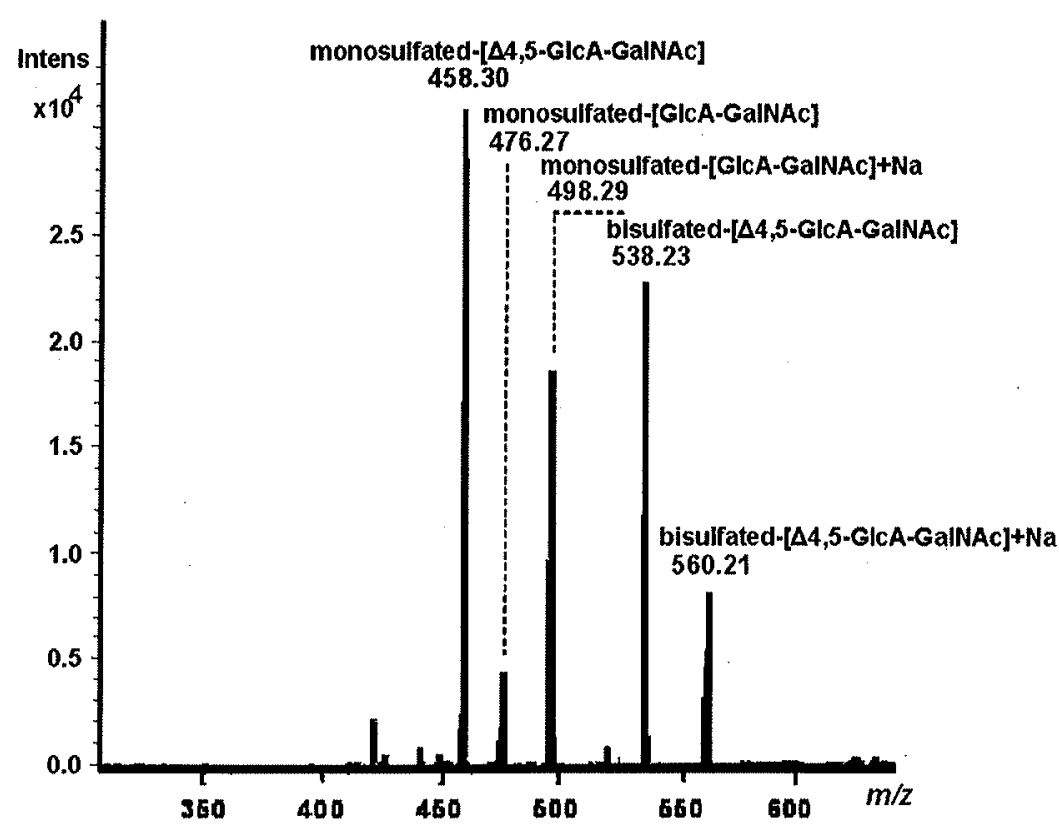


Fig. 3 Fully automated chip (-) nanoESI HCT CID $\mathrm{MS}^{2}$ of the singly deprotonated ion at $\mathrm{m} / \mathrm{z}$ 458.17 assigned according to mass calculation to monosulfated 4,5- $\Delta-$ [GlcA-GalNAc]. Diagnostic ions for GalNAc monosulfation are marked by filled diamonds. Inset structure of monosulfated CS disaccharide. $\mathrm{MS}^{2}$ by CID using He as a collision gas. Fragmentation amplitude within $0.40-0.80 \mathrm{~V}$. Other conditions as in Fig. 1

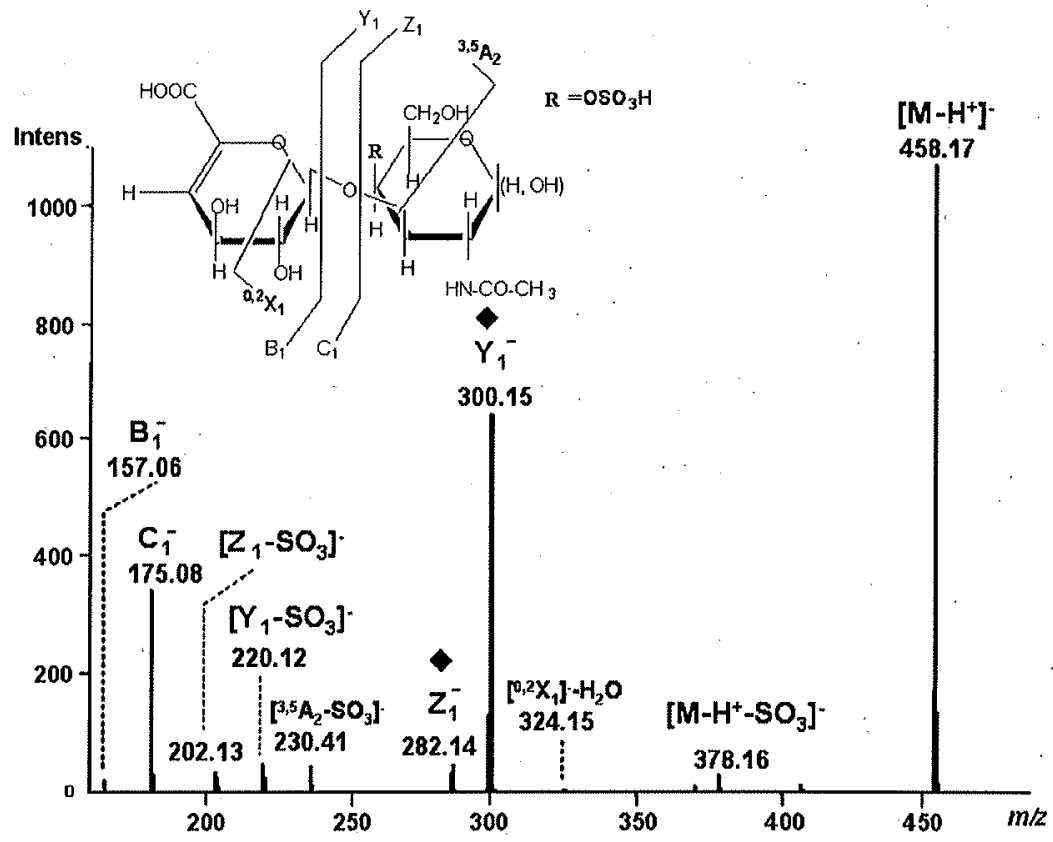

column (Amersham-Pharmacia, Freiburg, Germany), equilibrated and eluted in $150 \mu \mathrm{L}$ fractions with $0.5 \mathrm{M}\left(\mathrm{NH}_{4}\right)$ $\mathrm{HCO}_{3}$ at a flow rate of $0.5 \mathrm{~mL} / \mathrm{min}$ and continuous $\mathrm{UV}$ detection at $232 \mathrm{~nm}$. The column was calibrated with disaccharides obtained from recombinant expressed decorin digested with chondroitin ABC lyase [27]. The disaccharide fractions were pooled and desalted on a prepacked D-Salt column (MWCO 5000) (Pierce, Rockford, IL) [29].

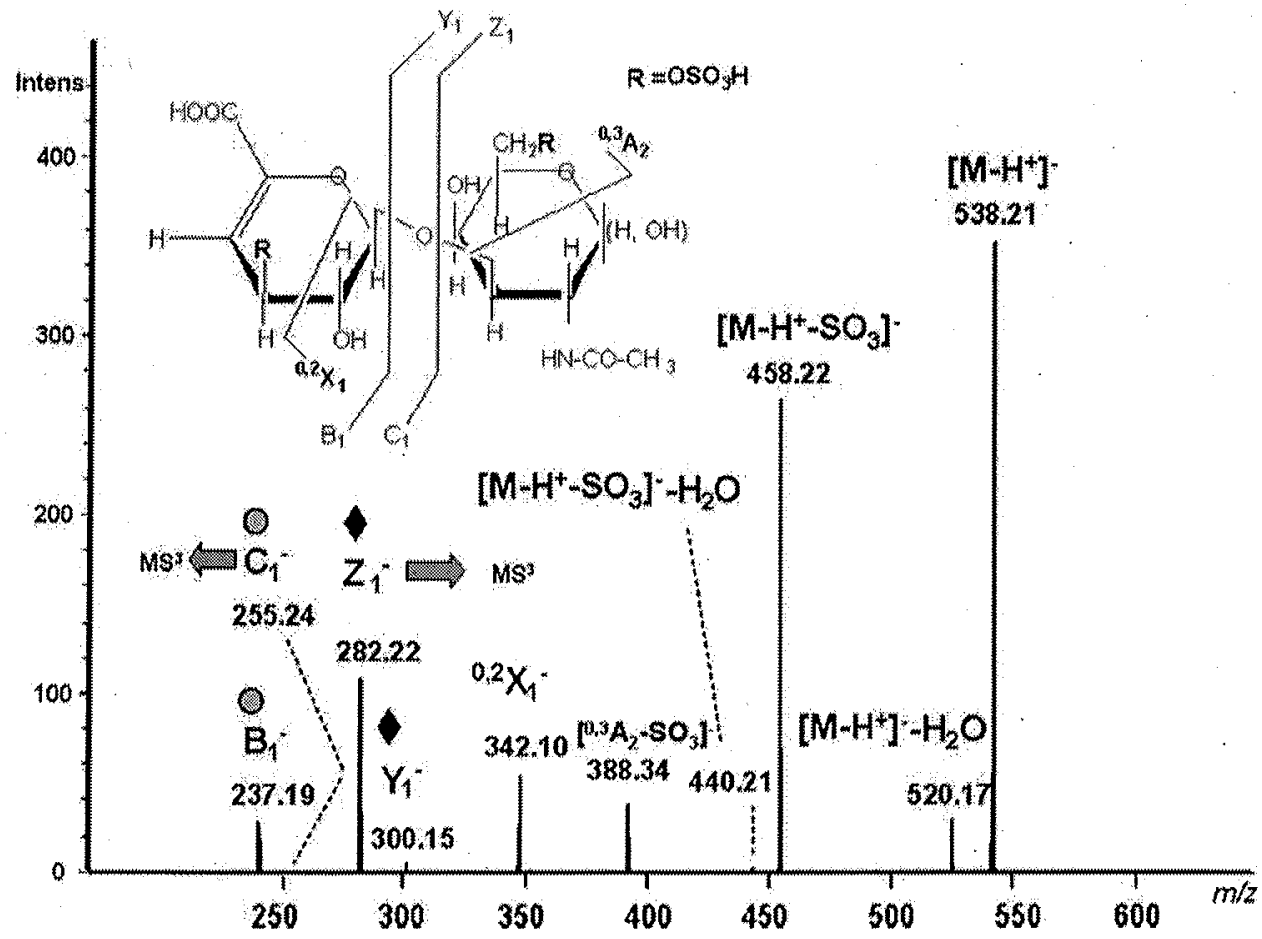

Fig. 4 Fully automated chip (-) nanoESI HCT CID MS ${ }^{2}$ of the singly deprotonated ions at $m / z 538.21$ assigned according to mass calculation to bisulfated 4,5- - -[GlcA-GalNAc]. Diagnostic ions for
GalNAc monosulfation are marked by filled diamonds; diagnostic ions for GlcA monosulfation are marked by filled circles. Inset structure of bisulfated CS disaccharide. Experiment conditions as in Fig. 3 
Mass spectrometry

Mass spectrometer All experiments were conducted on a High Capacity Ion Trap Ultra (HCT Ultra, PTM discovery) mass spectrometer from Bruker Daltonics, Bremen, Germany. All mass spectra were acquired in the mass range 100-1,000 $m / z$, with a scan speed of $8,000 \mathrm{~m} / \mathrm{zs}^{-1}$. Tandem mass spectrometry was carried out by collision-induced dissociation using $\mathrm{He}$ as the collision gas. For $\mathrm{MS}^{2}-\mathrm{MS}^{3}$ sequencing, the precursor ions were selected within an isolation width of $2 u$. Each fragmentation spectrum was obtained by accumulating scans at variable RF signal amplitudes within $0.40-0.80 \mathrm{~V}$, ramped from $30 \%$ to $200 \%$ within $40 \mathrm{~ms}$ per single spectrum and a fragmentation cutoff default of $27 \%$ of the precursor ion $m / z$.

The $m / z$ scale of the mass spectrum was calibrated by use of an external calibration standard G2421A electrospray "tuning mix" from Agilent Technologies (Santa Rosa, CA, USA). The reference provided in negative ion mode a spectrum with a fair ionic coverage of the $m / z$ range scanned in both MS and CID MS" experiments. The obtained mass accuracy was situated within 10-40 ppm, which is a normal range of an HCT MS instrument.
The assignment of molecular ions to a certain composition was made by exact mass calculation, taking as a base the previously acquired information $[22,24,30,31]$ and CS/DS biosynthesis pathway criteria. Fragment ion designation followed generally the published recommendations $[32,33]$.

Fully automated chip-based nanoelectrospray Samples were infused into the mass spectrometer using a fully automated chip-based nanoelectrospray system, NanoMate robot incorporating ESI 400 Chip technology (Advion BioSciences, Ithaca, USA). NanoMate is controlled and manipulated by ChipSoft 8.1 .0 software operating under Windows system. The robot was coupled to the HCT Ultra mass spectrometer via an in-laboratory made mounting system, which allows robot $\mathrm{O}$-xyz positioning with respect to HCT counterelectrode as described by us before $[31,34]$.

Five-microliter aliquots of the working sample solutions were loaded into the NanoMate 96-well plate. The robot was programmed to aspirate the whole volume of sample and afterwards deliver the sample to the inlet side of the 400 microchip. NanoMate HCT MS system was tuned for operating in the negative ion mode previously demonstrated

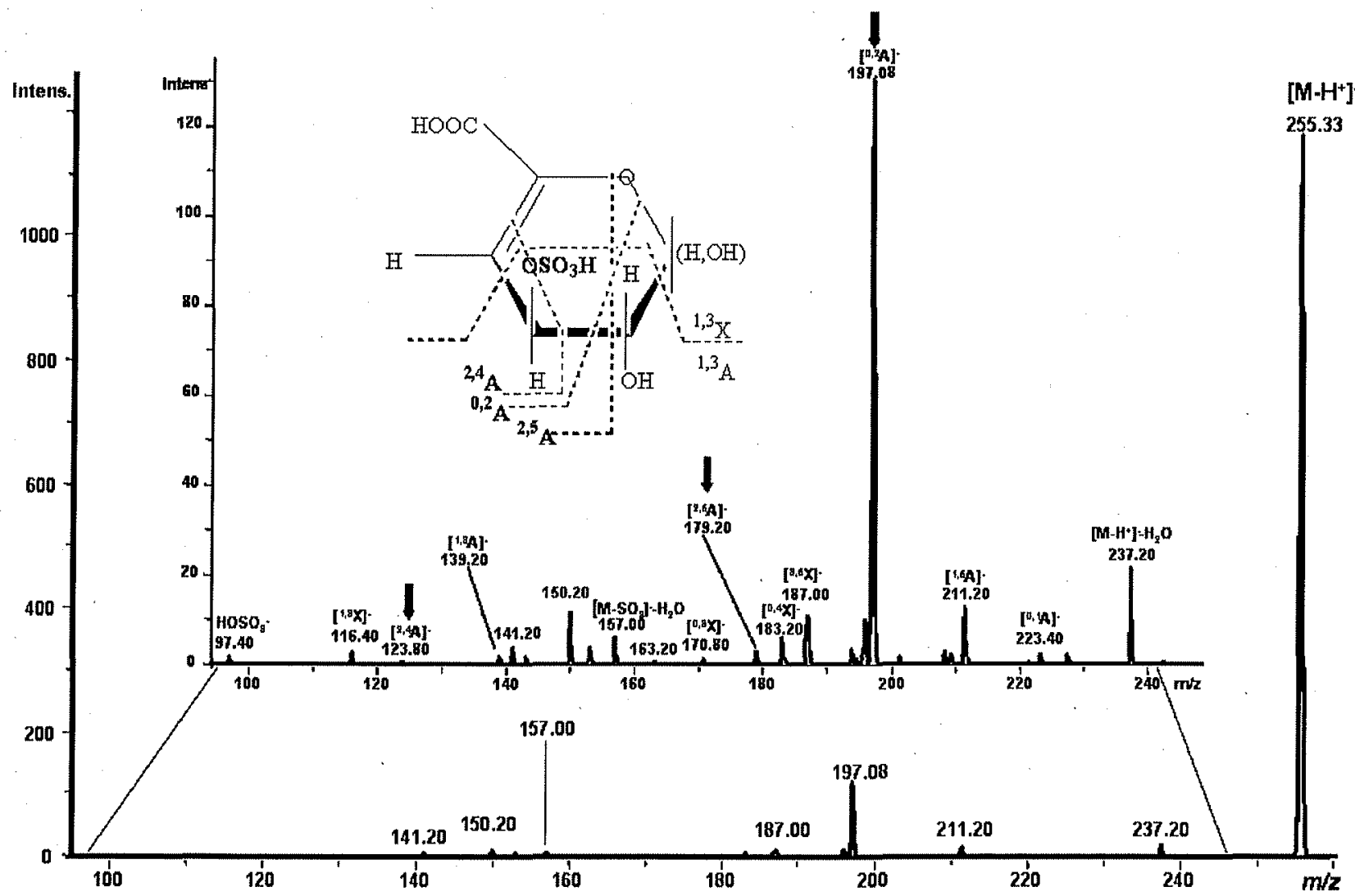

Fig. 5 Fully automated chip (-) nanoESI HCT CID MS ${ }^{3}$ of the $\mathrm{C}_{1}{ }^{-}$, fragment ion detected at $m / z 255.24$ in $\mathrm{MS}^{2}$. Inset zoomed $m / z(97-270)$ area and proposed ion structure. Diagnostic ions for GlcA (3S) is marked by arrow. Experiment conditions as in Fig. 3 
$[17,35,36]$ as the most appropriate ionization mode for glycosaminoglycans. Electrospray was initiated by applying a voltage of $-1.4 \mathrm{kV}$ on the pipette tip, $0.30 \mathrm{psi}$ nitrogen back pressure, and 50 psi pressure of nitrogen nebulizer on MS. In order to prevent possible in-source fragmentation, the potential difference between the end of the transfer capillary and the skimmer (Cap Exit) was set to $50 \mathrm{~V}$. The source block maintained at the constant temperature of $200{ }^{\circ} \mathrm{C}$ provided an optimal desolvation of the generated droplets without the need of desolvation gas. Following sample infusion and MS analysis, the pipette tip was ejected, and a new tip and nozzle were used for each sample, thus preventing any crosscontamination or carryover. Each chip nozzle had an internal diameter of $2.5 \mu \mathrm{m}$, which under the given conditions delivered a working flow rate of approximately $50 \mathrm{~nL} / \mathrm{min}$.

\section{Results and discussions}

Compositional analysis of brain DS and CS disaccharide fractions

Dermatan sulfate disaccharide fraction, obtained after depolymerization with chondroitin $B$ lyase dissolved in methanol, was loaded onto the microtiter plate of the
NanoMate robot and submitted to negative nanoESI chip for infusion and HCT MS analysis. Resulting DS mass spectrum as a sum of 60 scans acquired over $2 \mathrm{~min}$ is presented in Fig. 1. According to the mass calculation, the most abundant ion observed at $m / z 458.24$ was assigned a composition of monosulfated 4,5- $\Delta-$-[IdoA-GalNAc]. Such an assignment is based on the known specificity of chondroitin lyases. Chondroitin B lyase cleaves the glycosidic linkage between GalNAc and L-IdoA, while chondroitin AC I lyase cleaves the linkage between GalNAc and D-GlcA [28], with the formation of a 4,5 double bond at HexA and a water molecule elimination in both cases. These enzymes belong to the class of lyases that cleave glycosidic bond by $\beta$-elimination reaction, following this strict specificity, which does not depend on the sulfation content and/or pattern. Although after the action of the enzyme the stereochemistry is lost, in order to facilitate the discrimination between the uronic acids, we kept denoting them with their original IdoA and GlcA nomenclature. The mass spectrum in Fig. 1 is dominated by the singly charged ions at $\mathrm{m} / \mathrm{z} 458.24$ and 440.22 corresponding to monosulfated 4,5- $\Delta$-[IdoA-GalNAc]. Besides; MS screening indicates the presence of an unusual nonsulfated 4,5- $\Delta$ [IdoA-GalNAc] disaccharide visible as $\left[\mathrm{M}-\mathrm{H}^{-1}\right]^{-}$ion at $\mathrm{m} / \mathrm{z}$ 378.31 accompanied by the sodiated and dehydrated counterparts at $m / z 399.79$ and 360.29 , respectively.

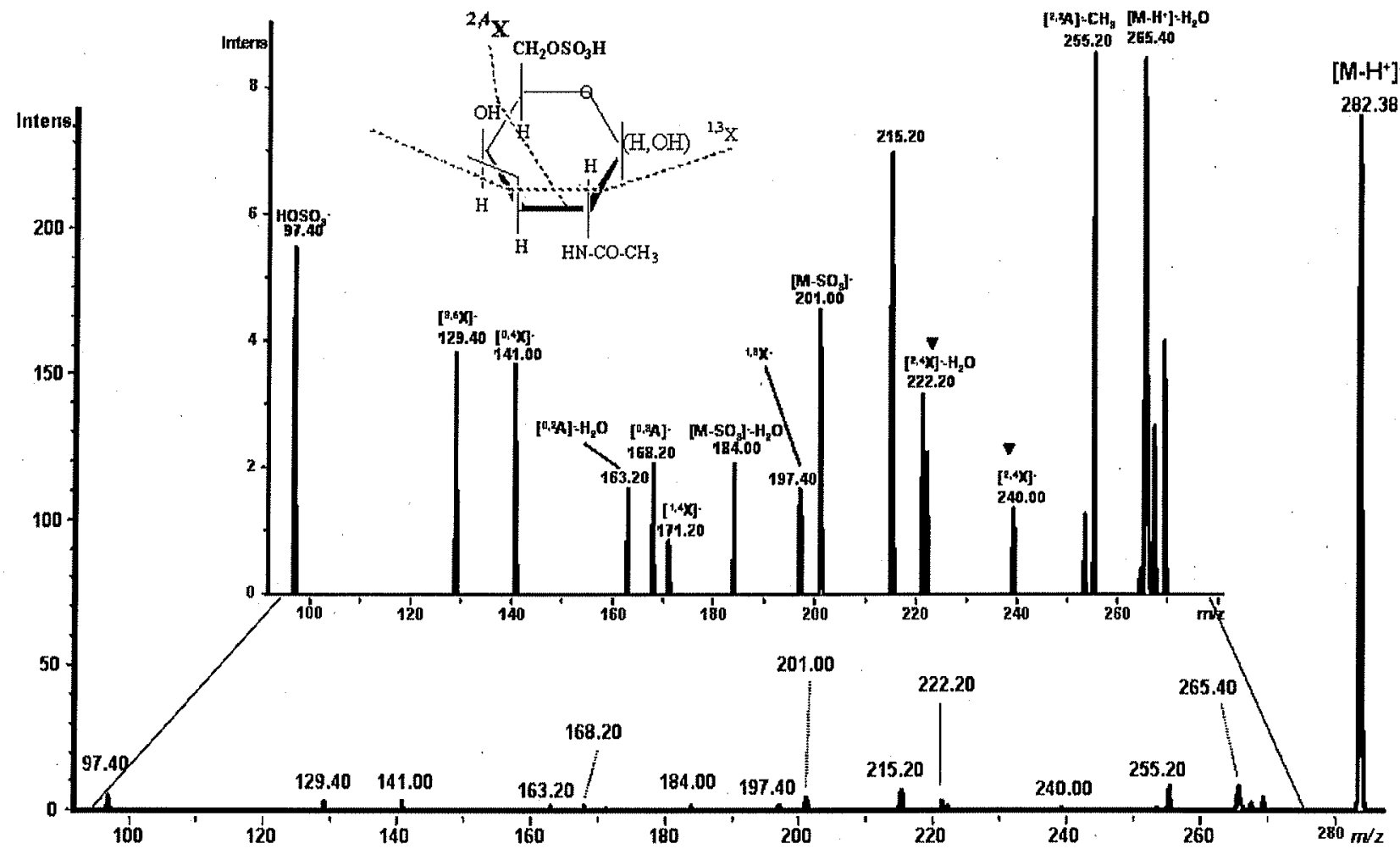

Fig. 6 Fully automated chip (-) nanoESI HCT CID MS ${ }^{3}$ of the $Z_{1}{ }^{-}$detected at $m / z 282.22$ in $\mathrm{MS}^{2}$. Diagnostic ions for GalNAc (6S) are marked by filled triangles. Inset zoomed $m / z(97-245)$ area and proposed ion structure. Experiment conditions as in Fig. 3 
MS analysis of disaccharide fraction obtained after depolymerization with AC I lyase rendered the spectrum presented in Fig. 2. In this spectrum, the most abundant ion at $\mathrm{m} / \mathrm{z} 458.30$ corresponds to a regularly monosulfated 4,5- $\Delta$-[GlcA-GalNAc] species, while the ions at $\mathrm{m} / \mathrm{z}$ 476.27 and $\mathrm{m} / \mathrm{z} 498.29$. disclose the presence of one irregular monosulfated [GlcA-GalNAc] compound lacking the C4-C5 double bond (Fig. 2). Most probably, this species represents the nonreducing end of the original hybrid CS/DS GAG chain. Additionally, the occurrence of a rare motif having the composition of bisulfated 4,5$\triangle$-[GlcA-GalNAc] is indicated by the $\left[\mathrm{M}-\mathrm{H}^{+}\right]$ion at $\mathrm{m} / \mathrm{z}$ 538.23 and $\left[\mathrm{M}-2 \mathrm{H}^{+}+\mathrm{Na}^{+}\right]$at $m / z$ 560.21. Unlike the disaccharide digested with $\mathrm{B}$ lyase, no unsulfated species were identified in the fraction obtained after depolymerization with AC I lyase.

\section{Determination of $\mathrm{CS}$ sulfation pattern by $\mathrm{CID} \mathrm{MS}^{2}-\mathrm{MS}^{3}$}

In the next stage of analysis, multistage $\mathrm{CID}\left(\mathrm{MS}^{2}-\mathrm{MS}^{3}\right)$ was employed for determination of mono- and oversulfa- tion patterns in disaccharide fraction obtained after depolymerization with chondroitin AC I lyase. Thus, the ion at $\mathrm{m} / \mathrm{z}$ 458.17 corresponding according to mass calculation to

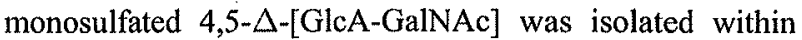
an isolation window of $2 \mathrm{u}$ and submitted to CID $\mathrm{MS}^{2}$. Obtained mass spectrum acquired over $60 \mathrm{~s}$ is presented in Fig. 3. As visible, employed optimized sequencing conditions enhanced the formation of sulfated fragment ions diagnostic for sulfate localization within the disaccharide. The ions at $\mathrm{m} / \mathrm{z} 282.14$ and 300.15 assigned according to Domon and Costello nomenclature [32] to $\mathrm{Z}_{1}{ }^{-}$and $\mathrm{Y}_{1}{ }^{-}$ result after GlcA-GalNAc glycosidic bond cleavage; they correspond to monosulfated GalNAc and therefore are diagnostic for the localization of the sulfate group at this monosaccharide residue. GalNAc monosulfation is proved also by the ring cleavage ion at $m / z 324.15$ assigned to $\left[{ }^{0,2} \mathrm{X}_{1}\right]-\mathrm{H}_{2} \mathrm{O} . \mathrm{B}_{1}^{-}$and $\mathrm{C}_{1}{ }^{-}$ions at $m / z \quad 157.06$ and 175.08 corresponding to nonsulfated GlcA bring additional evidence in support of this concept (Fig. 3).

In Fig. 4, fragmentation analysis by $\mathrm{CID} \mathrm{MS}^{2}$ of oversulfated $4,5-\Delta-[\mathrm{GlcA}-\mathrm{GalNAc}]$ disaccharide bearing two

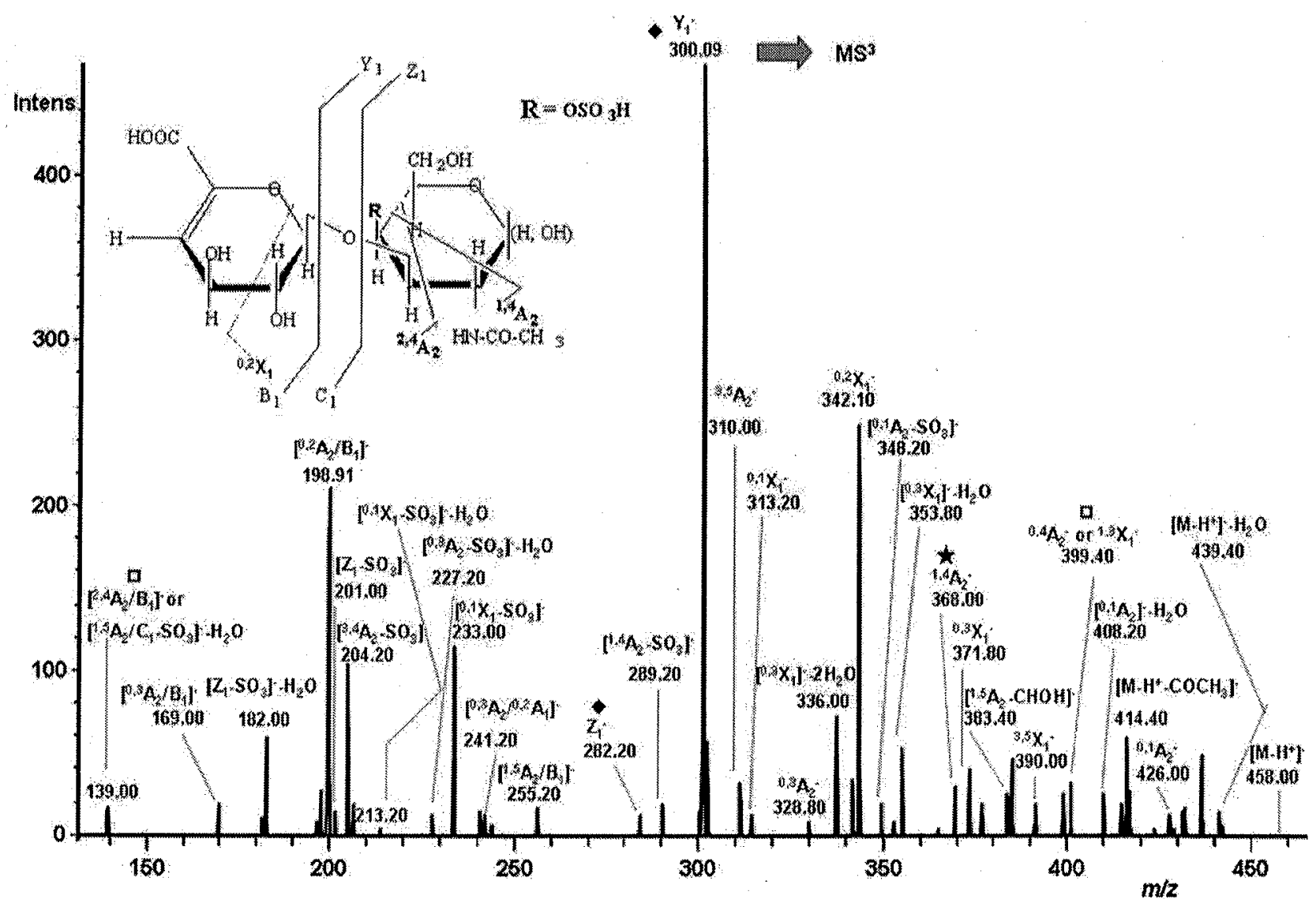

Fig. 7 Fully automated chip (-) nanoESI HCT CID MS ${ }^{2}$ of the singly deprotonated ions at $\mathrm{m} / \mathrm{z} 458.00$ assigned according to mass calculation to monosulfated 4,5- $\Delta$-[IdoA-GalNAc]. Diagnostic ions for GalNAc monosulfation are marked by filled diamond; diagnostic ion for GalNAc (4S) is marked by a star; possible diagnostic tons for GalNAc (4S) are marked by empty squares. Inset structure of monosulfated DS disaccharide. Experiment conditions as in Fig. 3 
$\mathrm{SO}_{3}{ }^{-}$groups is presented. This mass spectrum acquired also for $60 \mathrm{~s}$ exhibits a number of fragment ions corresponding to an unusual disaccharide structure. $\mathrm{Z}_{1}^{-}$and $\mathrm{Y}_{1}^{-}$ions at $\mathrm{m} / \mathrm{z}$ 282.22 and 300.15 as well as ${ }^{0,2} \mathrm{X}_{1}^{-}$at $m / z 342.10$ indicate that one sulfate group is localized at GalNAc, while $B_{1}^{-}$and $\mathrm{C}_{\mathrm{I}}^{-}$ions at $m / z 237.19$ and 255.24 are diagnostic for 4,5- $\Delta$ GlcA monosulfation. Consequently, the precursor ion corresponds to a bisulfated $4,5-\Delta$-[GlcA-GalNAc] structure in which each monosaccharide residue bears one sulfate ester group. Certainly, for this atypical species, unambiguous identification of sulfation sites at both monomer rings is of high biological significance. To identify the exact position of these sulfates within 4,5- $\Delta$-GlcA and GalNAc moieties of the investigated oversulfated disaccharides species, the ions at $\mathrm{m} / \mathrm{z} 255.24$ corresponding to $4,5-\Delta$ GlcA and $m / z 282.22$ corresponding to monosulfated GalNAc were submitted to fragmentation analysis in CID $\mathrm{MS}^{3}$ experiments.

Figure 5 presents CID $\mathrm{MS}^{3}$ of the ion at $m / z 255.24$ corresponding to monosulfated 4,5- $\triangle$-GlcA detected as $\mathrm{C}_{1}{ }^{-}$ fragment in $\mathrm{MS}^{2}$. Three ring cleavage ions diagnostic for an unusual position of sulfate within the monomer ring were detected. Thus, ${ }^{2,4} \mathrm{~A}^{-}$ion at $m / z 123.80,{ }^{2,5} \mathrm{~A}^{-}$ion at $m / z \quad 179: 20$, and ${ }^{0,2} \mathrm{~A}^{-}$ion at $m / z 197.08$ indicate that the sulfate group is located at position 3 of the $4,5-\Delta$-GlcA ring (inset Fig. 5).

$\mathrm{CID} \mathrm{MS}^{3}$ of the ion at $\mathrm{m} / \mathrm{z} 282.22$ corresponding according to mass calculation to monosulfated GalNAc detected as a $\mathrm{Z}_{1}^{-}$fragment ion in $\mathrm{MS}^{2}$ is depicted in Fig. 6. Inspection of the spectrum reveals that optimized fragmentation conditions in terms of RF signal amplitudes enhanced the formation of ring cleavage ions highly useful for sulfation site assessment. In this regard, the most relevant are ${ }^{2,4} \mathrm{X}^{-}$ion at $m / z 240.00$ and its dehydrated form $\left[{ }^{2,4} \mathrm{~A}\right]^{-}$$\mathrm{H}_{2} \mathrm{O}$ ion at $m / z 222.20$, which support a structure having the sulfation site located in position 6 of the GalNAc ring. This concept is additionally confirmed by the higher relative abundance of $\mathrm{Z}_{1}{ }^{-}$ion in comparison to that of $\mathrm{Y}_{1}{ }^{-}$ion. According to previous investigations [37, 38], such a distribution of signal relative intensities is consistent with GalNAc (6S) structure.

\section{Determination of DS sulfation pattern by CID $\mathrm{MS}^{2}-\mathrm{MS}^{3}$}

Detailed structural information including the localization of sulfation site at the monosulfated unsaturated 4,5- $\Delta-[$ IdoA-

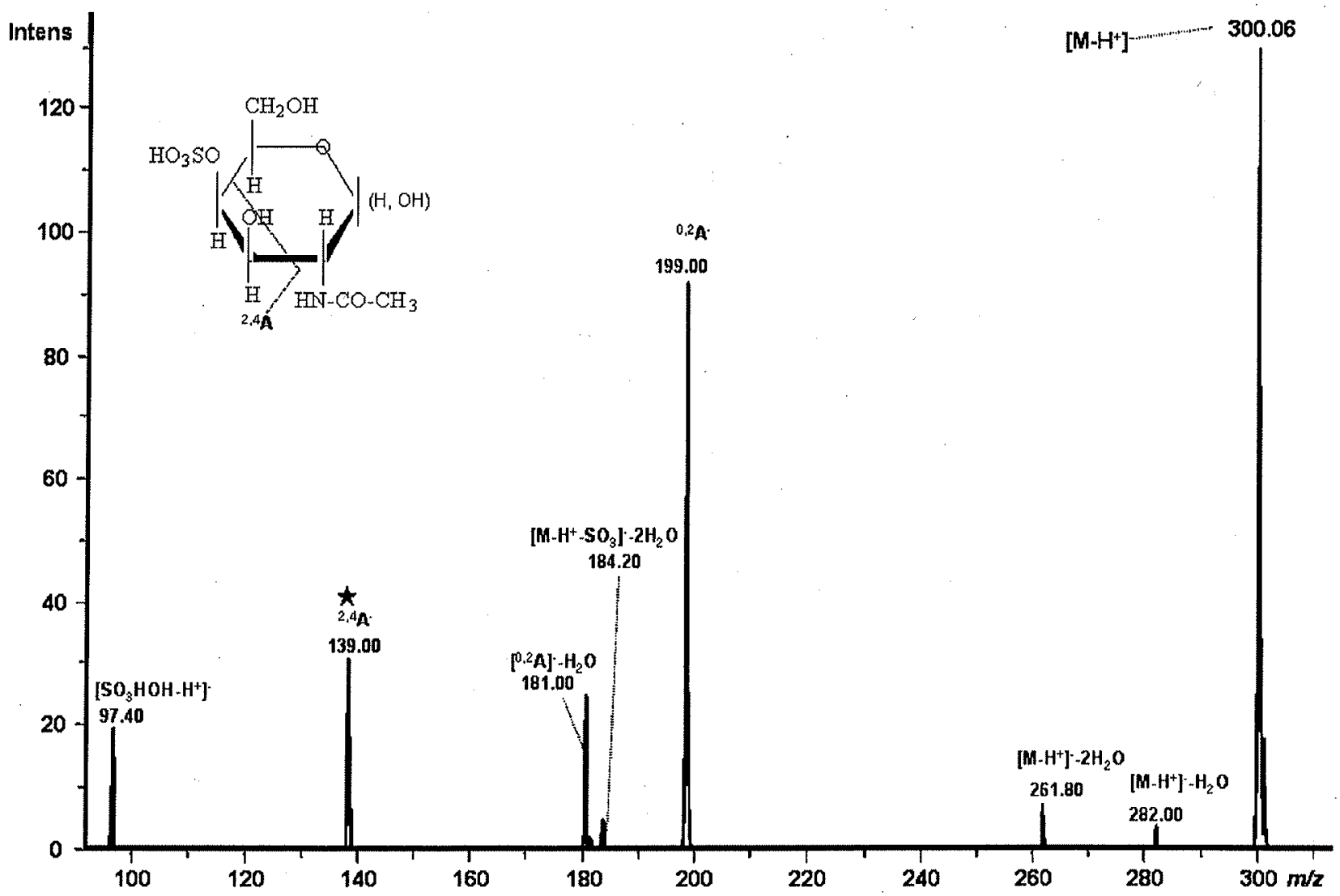

Fig. 8 Fully automated chip (-) nanoESI HCT CID MS ${ }^{3}$ of the $\mathrm{Y}_{1}{ }^{-}$ion detected at $m / z 300.09$ in $\mathrm{MS}^{2}$. Diagnostic ions for GalNAc (4S) are marked by a star. Inset structure of GalNAc (4S). Experiment conditions as in Fig. 3 
GalNAc] disaccharide detected as singly charged ion at $m / z$ 458.24 in MS was obtained by multistage CID $\mathrm{MS}^{2}-\mathrm{MS}^{3}$. CID $\mathrm{MS}^{2}$ fragmentation of the ion at $m / z 458.24$ resulted in the spectrum presented in Fig. 7. $\mathrm{Z}_{1}^{-}$and $\mathrm{Y}_{1}^{-}$as well as the ring cleavage ion ${ }^{0,1} \mathrm{X}_{1}^{-}$are diagnostic for the localization of sulfate group at GalNAc. ${ }^{1,4} \mathrm{~A}_{2}{ }^{-}$. ring cleavage ion marked by a star supports a structure in which the sulfate ester group is situated in position 4 of the GalNAc ring. Two additional ions marked in Fig. 7 by empty squares might corroborate this sulfation site. The ion at $\mathrm{m} / \mathrm{z} 139.00$ is assignable to either $\left[{ }^{2,4} \mathrm{~A}_{2} / \mathrm{B}_{1}\right]^{-}$or $\left[{ }^{1,5} \mathrm{~A}_{2} / \mathrm{C}_{1}-\mathrm{SO}_{3}\right]^{-}-\mathrm{H}_{2} \mathrm{O}$, while the ion at $m / z 399.40$ is consistent to either ${ }^{0,4} \mathrm{~A}_{2}{ }^{-}$or ${ }^{1,3} \mathrm{X}_{1}{ }^{-}$. $\left[{ }^{2,4} \mathrm{~A}_{2} / \mathrm{B}_{1}\right]^{-}$and ${ }^{0,4} \mathrm{~A}_{2}^{-}$ion fragment variants corroborate for the occurrence of sulfation in position 4 . To bring unequivocal evidence upon sulfate position at GalNAc, the ion at $\mathrm{m} / \mathrm{z}$ 300.09 , identified as an $\mathrm{Y}_{1}^{-}$in $\mathrm{MS}^{2}$ experiment, was isolated and submitted to $\mathrm{CID} \mathrm{MS}{ }^{3}$. Obtained mass spectrum is presented in Fig. 8. Obviously, optimized fragmentation conditions favored the formation of three-ring cleavage ions assigned to ${ }^{0,2} \mathrm{~A}^{-},{ }^{0,2} \mathrm{~A}^{-}-\mathrm{H}_{2} \mathrm{O}$, and ${ }^{2,4} \mathrm{~A}^{-}$. The latter one marked by a star is unambiguously diagnostic for sulfation in position 4 of GalNAc, a conclusion supported also by the higher intensity of the signal corresponding to $\mathrm{Y}_{1}^{-}$ion in comparison with that of $\mathrm{Z}_{1}^{-}$ion.

\section{Conclusions}

The aim of this study was to assess for the first time the feasibility of mass spectrometry in glycomics of brain glycosaminoglycans. For this purpose, an advanced, highly sensitive and rapid MS method based on fully automated chip (-) nanoESI HCT CID MS" was optimized and applied to CS and DS oligosaccharides extracted and purified from wild-type mouse brain. Chip-based nanoESI MS screening in the negative ion mode allowed for the identification of CS and DS disaccharide species of regular sulfation patterns as well as an unusual bisulfated CS motif. Detailed structural characterization and determination of sulfation sites were achieved by CID $\mathrm{MS}^{2}-\mathrm{MS}^{3}$ fragmentation. While CID MS/MS of regular and undersulfated GAGs was successfully performed before [29, 33, 35], MS/MS analysis of oversulfated domains [12-14, 19] did not allow an unequivocal determination of oversulfation sites. In a previous study conducted by CID MS/MS [12], although the sulfation of L-IdoA could be demonstrated, clear-cut discrimination between the structural motifs with either bisulfated D-GalNAc or monosulfated D-GlcA could not be achieved [26]. To overcome this limitation of single-stage CID fragmentation of oversulfated GAGs, we have recently developed [24] a methodology based on enzyme cleavage and multistage CID experiments. Application of CID MS ${ }^{\mathrm{n}}$ based protocol to oversulfated $\mathrm{CS} / \mathrm{DS}$ hexamers from human decorin led to the first unequivocal determination of excess sulfation sites along the GAG chain. In the present study, this novel protocol for oversulfated GAG analysis was for the first time optimized for CS/DS species expressed in CNS. By this method, novel 4,5- $\Delta-[\mathrm{GlcA}(3 \mathrm{~S})-$ GalNAc(6S)] and 4,5- $\Delta$-[IdoA-GalNAc(4S)] motifs were discovered and characterized. The presence of $4,5-\Delta-[\mathrm{GlcA}$ (3S)-GalNAc(6S)] confirmed here for the first time by chipbased nanoESI CID $\mathrm{MS}^{n}$ appears to corroborate the existence of the unusual GlcA 3-O-sulfotransferase reported in a few studies [39-41]. Nevertheless, the distribution of GlcA (3S) and sequences adjacent to GlcA (3S) in CS polymer chains remains to date less understood. Since several reports postulated that 3-O-sulfation might be involved or even trigger CS chain termination [39, 42], we suspect that the identified $4,5-\Delta-[\mathrm{GlcA}(3 \mathrm{~S})$-GalNAc (6S)] could be located close to the GAG chain extremity. Although such terminal motifs have not yet been confirmed, our findings appear to support the assumptions regarding their occurrence in CNS [43].

The methodology presented here is adaptable to all categories of glycosaminoglycans expressed in central nervous system and is likely to become further improved in terms of application to longer CNS GAG chains and extension towards GAG species associated to human brain in health and disease. Method straightforward application to research oriented towards elucidation of CS/DS sulfation role in brain development, maturation, and aging as well as various CNS pathologies is envisaged. Moreover, further studies focused on quantifying sulfation patterns in development or disease using combined liquid chromatographychip-based nanoESI CID MS ${ }^{n}$ are deemed necessary and therefore planned.

Acknowledgments We are grateful to Prof. Dr. Ecaterina Andronescu, Romanian Minister of Education, Research and Innovation, and Prof. Dr. Lizica Mihut, Rector of "Aurel Vlaicu" University of Arad, Romania, for their invaluable support.

This work was supported by Romanian National Authority for Scientific Research through the grants CE.EX. 111/2006, 98/2006, and PN-II-41001/2007 and German Society for Research (grant SFB 492/A9 and DFG SE1431/1-1).

\section{References}

1. Sherman LS, Back SAA (2007) Trends Neurosci 31:44-52

2. Seidler DG, Dreier R (2008) IUBMB Life 60:729-733

3. Lyon M, Deakin JA, Rahmoune H, Fernig DG, Nakamura T, Gallagher JT (1998) J Biol Chem 273:271-278

4. Sugahara K, Mikami T, Uyama T, Mizuguchi S, Nomura K, Kitagawa H (2003) Curr Opin Struct Biol 13:612-620

5. Trowbridge JM, Rudisill JA, Ron D, Gallo RL (2002) J Biol Chem 277:42815-42820

6. Crespo D, Asher RA, Lin R, Rhodes KE, Fawcett JW (2007) Exp Neurol 206:159-171 
7. Bovolenta P, Fernaud-Espinosa I (2000) Progr Neurobiol 61:113-132

8. Purushothaman A, Fukuda J, Mizumoto S, ten Dam GB, van Kuppevelt TH, Kitagawa H, Mikami T, Sugahara KJ (2007) J Biol Chem 282:19442-19452

9. Bao X, Pavao MSG, dos Santos JC, Sugahara K (2005) J Biol Chem 280:23184-23193

10. Bao X, Muramatsu T, Sugahara K (2005) J Biol Chem $280: 35318-35328$

11. Hikino M, Mikami T, Faissner A, Vilela-Silva ACES, Pavao MSG, Sugahara K (2003) J Biol Chem 278:43744-43754

12. Zamfir A, Seidler DG, Kresse H, Peter-Katalinic J (2003) Glycobiology 13:733-742

13. Zamfïr A, Seidler DG, Schönherr E, Kresse H, Peter-Katalinic J (2004) Electrophoresis 25:2010-2016

14. Zamfir A, Peter-Katalinić J (2004) Electrophoresis 25:1949-1963

15. Miller MJC, Costello CE, Malmström A, Zaia J (2006) Glycobiology 16:502-513

16. Saad OM, Leary JA (2005) Anal Chem 77;5902-5911

17. Saad OM, Myers RA, Castleton DL, Leary JA (2005) Anal Biochem 344:232-239

18. Saad OM, Ebel H, Uchimura K, Rosen SD, Bertozzi CR, Leary JA (2005) Glycobiology 15:818-826

19. Mormann M, Zamfir AD, Seidler DG, Kresse H, Peter-Katalinic J (2007) J Am Soc Mass Spectrom 18:179-187

20. Wolff JJ, Laremore TN, Aslam H, Linhardt RJ, Amster IJ (2008) J Am Soc Mass Spectrom 19:1449-1458

21. Wolff JJ, Laremore TN, Busch AM, Linhardt RJ, Amster IJ (2008) J Am Soc Mass Spectrom 19:294-304

22. Seidler DG, Peter-Katalinić J, Zamfir AD (2007) Sci World J 7:233-241

23. Amon S, Zamfir AD, Rizzi A (2008) Electrophoresis 29:2485-2507

24. Zamfir AD, Flangea C, Serb AF, Sisu E, Dinca N, Bruckner P, Seidler DG (2009) Proteomics 9:3435-3444

25. Zamfir AD, Bindila L, Lion N, Allen M, Girault HH, PeterKatalinic J (2005) Electrophoresis 26:3650-3673
26. Zamfir A, Vakhrushev S, Sterling A, Niebel H, Allen M, PeterKatalinić J (2004) Anal Chem 76:2046-2054

27. Seidler DG, Breuer E, Grande-Allen KJ, Hascall VC, Kresse H (2002) J Biol Chem 277:42409-42416

28. Gu K, Linhardt RJ, Laliberte M, Gu K, Zimmermann J (1995) Biochem J 312:569-577

29. Zamfir A, Seidler DG, Kresse H, Peter-Katalinc J (2002) Rapid Commun Mass Spectrom 16:2015-2024

30. Zaia J (2004) Mass Spectrom Rev 23:161-227

31. Flangea C, Serb AF, Schiopu C, Tudor S, Sisu E, Seidler DG, Zamfir AD (2009) Cent Eur J Chem (in press)

32. Domon B, Costello CE (1988) Glycoconj J 5:397-409

33. Zaia J, McClellan JE, Costello CE (2001) Anal Chem 73:6030 6039

34. Almeida R, Mosoarca C, Chirita M, Udrescu V, Dinca N, Vukelic $\mathrm{Z}$, Allen M, Zamfir AD (2008) Anal Biochem 378:43-52

35. Zaia J, Miller MJ, Seymour JL, Costello CE (2007) J Am Soc Mass Spectrom 18:952-960

36. Chi L, Munoz EM, Choi HS, Ha YW, Kim YS, Toida T, Linhardt RJ (2006) Carbohydr Res 341:864-869

37. Desaire H, Leary J (2000) J Am Soc Mass Spectrom 11:916-920

38. Linhardt RJ, Wang HM, Loganthan D, Lamb DJ, Mallis LM (1992) Carbohydr Res 225:137-145

39. Kinoshita A, Yamada S, Haslam SM, Morris HR, Dell A, Sugahara K (2001) Biochemistry 40:12654-12665

40. Kinoshita A, Yamada S, Haslam SM, Morris HR, Dell A, Sugahara K (1997) J Biol Chem 272:19656-19665

41. Kinoshita-Toyoda A, Yamada S, Haslam SM, Khoo KH, Sugiura M, Morris HR, Dell A, Sugahara K (2004) Biochemistry 43:11063-11074

42. Kitagawa $\mathrm{H}$, Tsutsumi $\mathrm{K}$, Ujikawa $\mathrm{M}$, Goto $\mathrm{F}$, Tamura $\mathrm{J}$, Neumann KW, Ogawa T, Sugahara K (1997) Glycobiology $7: 531-537$

43. Allory $\mathrm{Y}$, Commo F, Boccon-Gibod L, Sibony $M$, Callard $P$, Ronco P, Debiec H (2006) J Histochem Cytochem 54:575-584 